\title{
Enhancement of Phosphorus Activation in Vacancy Engineered thin Silicon-on-Insulator Substrates
}

\author{
A J Smith*, R M Gwilliam and V Stolojan \\ Ion Beam Centre, Advanced Technology Institute, University of Surrey, Guildford, \\ GU2 7XH, United Kingdom. \\ A P Knights \\ Department of Engineering Physics, McMaster University, 1280 Main Street West, \\ Hamilton, Ontario L8S 4L7, Canada. \\ P G Coleman and A Kallis \\ Department of Physics, University of Bath, Bath, BA2 7AY, United Kingdom. \\ S. H. Yeong \\ Dept. of Chemical and Biomolecular Eng., National University of Singapore, 9 \\ Engineering Drive 1, 1175776, Singapore \\ * Corresponding author: a.j.smith@ surrey.ac.uk \\ $+441483682144$
}

PACs: 61.80.Jh, 61.72.U-, 61.72.J-, 61.72.jd, 61.72.Cc 


\section{Abstract}

The concentration of vacancy-type defects in a silicon-on-insulator substrate consisting of a $110 \mathrm{~nm}$ silicon overlayer and $200 \mathrm{~nm}$ buried oxide has been quantified using Variable Energy Positron Annihilation Spectroscopy following 300keV $1.5 \times 10^{15} \mathrm{~cm}^{-2} \mathrm{Si}^{+}$ion implantation and subsequent annealing at temperatures ranging from $300-700^{\circ} \mathrm{C}$. The preferential creation of vacancies (relative to interstitials) in the silicon overlayer leads to a net vacancy-type defect concentration after annealing. Assuming that the defects have a structure close to that of the divacancy we determine the concentration to range from $1.7 \times 10^{19}-5 \times 10^{18} \mathrm{~cm}^{-3}$ for annealing temperatures of $300-700^{\circ} \mathrm{C}$. The measured concentration is in excellent agreement with that predicted via Monte-Carlo simulation. The impact of this net vacancy population on the diffusion and activation of phosphorus introduced by a $2 \mathrm{keV}$ implantation to a dose of $1 \times 10^{15} \mathrm{~cm}^{-2}$ has been observed. For samples which combine both $\mathrm{Si}^{+}$and $\mathrm{P}^{+}$ implantation, post-implantation phosphorus diffusion is markedly decreased relative to that for $\mathrm{P}^{+}$implantation only. Further, a four-fold increase in the electrical activation of phosphorus after post-implantation annealing at $750^{\circ} \mathrm{C}$ is observed when both implantation of $\mathrm{Si}^{+}$and $\mathrm{P}^{+}$is performed. We ascribe this affect to the reduction of phosphorus - interstitial clusters by the excess vacancy concentration beyond the amorphous/crystalline interface created by the $\mathrm{P}^{+}$implantation. 


\section{Introduction}

As Complementary-Metal-Oxide-Semiconductor (CMOS) devices evolve beyond the $32 \mathrm{~nm}$ technology node, the device requirements push the fundamental boundaries of not only the starting substrate, namely silicon, but also the technology used to create such advanced devices [1]. Source/Drain Extensions (SDE) are vital components within the device architecture, helping reduce detrimental phenomena such as short channel effects. However, the ability to produce such Ultra-Shallow Junctions (USJs) to the required specification in terms of sheet resistance $\left(R_{s}\right)$ and junction depth $\left(X_{j}\right)$ with a traditional implantation and anneal process, is significantly reduced due to dopant-defect interactions which result in anomalous diffusion [2] and clustering behavior [3] that increases the junction depth and sheet resistance, respectively.

Vacancy engineering is a co-implantation process whereby an excess of vacancy defects, the concentration of which is often estimated via simulation, are first generated in the region of the required shallow junction in the expectation that the excess vacancies will annihilate the supersaturation of interstitials introduced during the subsequent dopant implant [4]. This minimizes the unfavorable effect of excess defects on interstitial mediated dopants such as boron. By combining a Vacancy Engineering Implantation (VEI) with a Silicon-on-Insulator (SOI) substrate it is possible to optimize the introduction of vacancies by implanting at an energy which ensures the ion species resides beyond the buried oxide, thus leaving the peak of the interstitial distribution within the underlying substrate [5]. Such an implantation process provides an excess of vacancy defects in the SOI overlayer with the result that the two excess defect bands (interstitial and vacancy) are physically isolated on either side of the buried oxide (BOX). A variation of this process was demonstrated recently, in which the co-implantation dose and energy was engineered to provide a 
buried amorphous layer encroaching into the top silicon over-layer, above the BOX [6]. This placed the subsequent doping implantation within a highly damaged but crystalline surface region, allowing the overlayer to repair thermally via a 'reverse' solid phase epitaxial regrowth.

The direct determination of the concentration and distribution of vacancy-type defects following ion implantation of silicon is non-trivial. Although several techniques have been utilized to this end, each is limited in the type, range or concentration of defects which can be probed. Variable Energy Positron Annihilation Spectroscopy (VEPAS) has been shown to provide semi-quantitative data on the formation and evolution of vacancy defects introduced via ion implantation [7], while in circumstances where the defect type may be assumed reliably determination of defect concentration is possible. In this report we use VEPAS to examine the concentration of excess vacancy defects generated by a VEI in a thin SOI layer. The experimentally-determined defect concentrations are compared to Monte Carlo simulations, and related to the efficiency for improving phosphorous activation and reducing transient enhanced diffusion.

\section{Experimental Method}

SOI wafers with a $110 \mathrm{~nm} / 200 \mathrm{~nm}$ structure (silicon overlayer/BOX thickness) were implanted with a $300 \mathrm{keV}$ silicon VEI to a dose of $1.5 \times 10^{15} \mathrm{~cm}^{-2}$, positioning the projected range, and therefore the excess interstitial region, beneath the BOX. The experimental design and estimation of the defect generation is presented within Fig. 1(a), illustrating the simulated excess vacancy (open squares) and interstitial (open circles) distributions with respect to the SOI structure. These were calculated using a Monte Carlo routine and the following expression: 


$$
C_{D}(x)=\left[S i_{I}(x)-S i_{V}(x)\right]+S i_{D}(x)
$$

where, $C_{D}(x)$ is the defect distribution calculated from $S i_{I}(x), \operatorname{Si}_{V}(x)$ and $S i_{D}(x)$ representing the silicon interstitial, vacancy concentration and silicon ion implanted distribution, respectively [8]. Therefore, if $C_{D}(x)$ is negative (positive) the defect distribution is determined to be vacancy-rich (interstitial-rich).

Complete amorphization of the SOI overlayer inhibits thermal regrowth of the overlayer into mono-crystalline silicon (poly-crystalline silicon is formed), and excess vacancies do not survive. Cross-sectional Transmission Electron Microscopy (XTEM) analysis was used to probe the damage sustained to the substrate after the VEI (Fig. 1(b - e)). Samples were prepared by mechanical grinding and polishing, followed by ion-beam polishing at $4.5^{\circ}$ in a Gatan Precision Ion Polishing System using Ar ions at $4.5 \mathrm{keV}$. The samples were imaged in a Hitachi HD2300A Scanning Transmission Electron Microscope at 200keV, with a Schottky emitter. Diffraction patterns were acquired using a small, parallel beam of $\sim 10 \mathrm{~nm}$ in diameter.

The diffraction pattern shown in Fig. 1(b) indicates that the silicon over-layer has a single crystal structure, confirming the VEI has not destroyed the overall crystalline nature of the silicon layer. The diffraction pattern in Fig. 1(c) is typical for an amorphous material and is thus consistent with a buried $\mathrm{SiO}_{2}$ amorphous layer, whilst 1(d) illustrates the diffraction pattern obtained from just below the BOX, around the peak of the interstitial damage region where most of the damage is expected. The rings are much sharper than in the case of 1(c), suggesting that the layer is nano-crystalline or has long-range ordering present. Lastly, 1(e) shows the diffraction pattern from beyond the implant distribution, illustrating a single crystal structure (similar to that for Fig. 1(b)). 
Once the crystalline integrity of the silicon over-layer after the VEI had been verified, samples were annealed using a 10 s isochronal scheme from 300 to $700^{\circ} \mathrm{C}$ prior to performing VEPAS at the University of Bath [9]. VEPAS data are represented using the $S$-parameter, which reflects the average momentum of electrons at the positron/electron annihilation sites. In general, an increase in the value of $S$, relative to that for a sample that is essentially defect free, indicates the presence of open-volume or vacancy-type defects. The amount by which $S$ increases reflects both the size and total concentration of the defects within the sample. The VEPAS technique is sensitive to defect concentrations as low as $10^{15} \mathrm{~cm}^{-3}$, while positron trapping saturates for samples containing defects at a concentration $>10^{19} \mathrm{~cm}^{-3}$. Control of the energy of the implanted positrons, typically between 0.5 and $30 \mathrm{keV}$, allows one to probe defect distributions from the sample surface to a few microns in depth. The positron implantation profile is well described by a Makhovian distribution:

$$
P(z, E)=-\frac{d}{d x}\left\{\exp \left[z / z_{0}\right]^{m}\right\}
$$

where $z$ is the distance measured from the surface, $E$ is the incident positron energy, and $z_{0}$ is related to the mean implantation depth $\bar{z}$ for a material of density $\rho$ and is commonly given by $(40 / \rho) E^{1.6}\left(z_{0}\right.$ in $\mathrm{nm}$ and $E$ in $\left.\mathrm{keV}\right)$.

\section{Results and Discussion}

Fig. 2 shows a selection of plots of $S$-parameter versus incident positron energy; $S$ values are normalized to that measured for single crystalline, low-doped silicon. Inspection of the plots provides useful qualitative information related to the atomic structure of the SOI samples. For the unimplanted sample, the $S$-parameter for energies consistent with the $100 \mathrm{~nm}$ overlayer (i.e. up to $3 \mathrm{keV}$ ) is between $0.93-0.94$. 
As shown previously, this is an indication of a relatively high quality thin silicon layer [10]. Positrons implanted into this layer diffuse either to the sample surface or the overlayer/BOX interface and thus the $S$-parameter is indicative of the $\mathrm{Si} / \mathrm{SiO}_{2}$ interface. For positron energies consistent with the BOX (i.e. 3-6keV), the $S$ parameter increases to $\sim 0.96$, indicative of $\mathrm{SiO}_{2}$. The $S$-parameter exhibits a monotonic increase for energies increasing above $6 \mathrm{keV}$, representative of diffusion from the silicon substrate to the BOX/substrate interface. At the highest positron energies the $S$-parameter approaches 1 , the value for undefected silicon. Following implantation (and subsequent thermal annealing) the $S$-parameter plots indicate the presence of open-volume (vacancy-type) defects in the silicon overlayer. This is manifested by an increase in the relevant $S$-parameter to a value $>1$. The defects themselves most probably have a specific $S$-parameter $\sim 1.04$ [11], although the width of the positron implantation profile prevents annihilation of all implanted positrons within the silicon overlayer for any energy.

The data presented in Fig. 2, and similar data for all other annealing temperatures, were modeled using the fitting routine POSTRAP [12]. The structures were assumed to possess seven annihilation sites: (1) the sample surface, (2) the silicon overlayer, (3) the silicon overlayer/BOX interface, (4) the BOX, (5) the BOX substrate interface, (6) the implanted substrate, and (7) the undefected substrate. The $\mathrm{SiO}_{2} / \mathrm{Si}$ interfaces associated with the BOX were assumed to possess a thickness of $7 \mathrm{~nm}$, a requirement of the fitting routine to enable the interfaces to act as perfect positron traps. The thickness of the overlayer was fixed at $110 \mathrm{~nm}$ in all fits. The implantation energy of the $\mathrm{Si}^{+}$ions was such that defects were created in the underlying silicon substrate. The range of these defects was fixed for all of the data presented here to be $800 \mathrm{~nm}$ from the sample surface. The surface $S$-parameter was 
fixed in a range varying from 0.94 to 0.96 for all models. The interface $S$-parameters associated with the BOX $\left(S_{\text {int }}\right)$ were allowed to vary in each model, but values for the front and back interface were always required to be the same as one another. For the undefected sample $S_{i n t}$ was 0.89 , whereas for the implanted and subsequently annealed samples $S_{i n t}$ was found to increase monotonically with annealing temperature from 0.91 for the implanted sample to 0.99 for the sample annealed at $700^{\circ} \mathrm{C}$. This variation is as much an indication of the decreased influence of the interfaces on positron trapping for defected samples as a physical change in the interface itself. The BOX layer, being amorphous with a small positron diffusion length, has an $S$-parameter which is indicative of the oxide positron trapping site. This was 0.98 for the unimplanted sample, being reduced to 0.91 after implantation and increasing monotonically with annealing temperature to a value of 0.98 after annealing at $700^{\circ} \mathrm{C}$. This behavior is consistent with that measured previously for ion implanted $\mathrm{SiO}_{2}[13]$.

The most significant result in the present work is the determination of the defect concentration in the silicon overlayer. By maintaining the values of $S$ parameter for the undefected silicon overlayer at 1.00 and the value of the defect trapping site in the overlayer and the silicon substrate at 1.04, it was possible to fit all of the data obtained in this study by varying the modeled defect concentration. The results of this fitting process are summarized in Fig. 3, where we plot the fitted defect concentration in the overlayer versus annealing temperature. Although there exist seven possible trapping sites for positrons in this system, through careful imposition of boundary conditions to the fitting procedure we were able to retain physically realistic models to represent each sample plot. 
Fig. 3 presents the measured concentration of vacancies within the $110 \mathrm{~nm}$ silicon surface layer, assuming a 'box-like' distribution, where the second $y$-axis illustrates the areal density of vacancies as a function of anneal temperature. The excess vacancy distribution estimated in Fig. 1 assumes a local recombination of the generated Frenkel pair population. By assuming that this occurs at around $300^{\circ} \mathrm{C}[14]$ it is possible to compare the areal density of vacancies measured by VEPAS with that estimated by the simulation, after doubling the former because the $S$ parameter observed here is associated with divacancies [11]. Then the measured density of $3.7 \times 10^{14} \mathrm{~cm}^{-2}$ is in very good agreement with the simulation result of $4.25 \times 10^{14} \mathrm{~cm}^{-2}$.

We now consider the effects of the VEI on a phosphorus-doped USJ. A second set of identical samples was implanted with the same specification VEI as for the VEPAS measurements described above. A subsequent phosphorus implantation was performed at an energy of $2 \mathrm{keV}$ and to a dose of $10^{15} \mathrm{~cm}^{-2}$. The samples were then subjected to a $10 \mathrm{~s}$ anneal at temperatures ranging from 600 to $1000^{\circ} \mathrm{C}$ in a $\mathrm{N}_{2}$ ambient. Evolution of the phosphorus dopant profile was determined via Secondary Ion Mass Spectroscopy (SIMS) while the electrical activation of the phosphorus was measured in terms of sheet resistance $\left(R_{S}\right)$ using a four point probe. Concomitant determination of resistivity and mobility were measured via the Hall effect using the Van der Pauw principle and geometry. The results are presented in Figs. 4 and 5.

For the sample set without a VEI (Fig. 4(a)), a significant degree of diffusion is apparent within the tail region of the phosphorus distribution when the anneal temperature is increased to above $700^{\circ} \mathrm{C}$. When a VEI is used (Fig. 4(b)) annealing below $900^{\circ} \mathrm{C}$ results in a reduction in diffusion (compared to the sample set which did not receive a VEI) is observed, resulting in a more abrupt phosphorus profile. 
This indication that phosphorus transient enhanced diffusion is reduced significantly when the VEI is used is consistent with phosphorus being an interstitial diffuser. It is interesting to note that after post phosphorus implantation annealing at $800^{\circ} \mathrm{C}$ without a VEI, a 'kink' appears in the phosphorus profile at a concentration of approximately $10^{19} \mathrm{~cm}^{-3}$. For shallow boron junction formation such a kink is often associated with the level of dopant electrical activation. In the case of phosphorus, as shown in Fig. 4, the 'kink' point occurs at a significantly higher concentration when a VEI is used compared to when it is absent.

Examination of the $R_{s}$ of the activated phosphorus doped layer without a VEI as a function of anneal temperature (shown in Fig. 5 as open circles) reveals a distinct deactivation/reactivation process which occurs between $700^{\circ} \mathrm{C}$ and $900^{\circ} \mathrm{C}$. However, when a VEI is used (open squares), no deactivation is present even though the $R_{S}$ is initially higher at low temperatures. Moreover, within the $750^{\circ} \mathrm{C}-850^{\circ} \mathrm{C}$ temperature range the $R_{s}$ is consistently lower, resulting in a peak $R_{s}$ improvement of approximately $35 \%$ at $850^{\circ} \mathrm{C}$. To gain a further understanding of the $R_{s}$ trends, Hall measurements were performed and the results are shown in Figs. 5 (b) and (c). Without a VEI the deactivation process between $700^{\circ} \mathrm{C}$ and $750^{\circ} \mathrm{C}$ results in an initial reduction of the carrier density while the carrier mobility increases due to a reduction in the ionized impurity scattering. As the anneal temperature is increased towards $900^{\circ} \mathrm{C}$ the carrier density increases causing the mobility to decrease monotonically.

When a VEI is used the carrier density is consistently higher throughout the $600^{\circ} \mathrm{C}$ to $900^{\circ} \mathrm{C}$ temperature range and the reduction observed between $700^{\circ} \mathrm{C}$ and $800^{\circ} \mathrm{C}$ is significantly less than when a VEI is not used. However, the relative increase in $R_{\mathrm{s}}$ between $600^{\circ} \mathrm{C}$ and $700^{\circ} \mathrm{C}$ for the case of VEI is thought to be due to a reduction in the carrier mobility, a result of residual implantation damage. Above 
$900^{\circ} \mathrm{C}$ the effect of the VEI diminishes in a manner very similar to previous studies on boron doping [15].

Phosphorus has previously been shown to cluster with vacancy defects [16]. Therefore, one may reasonably expect that the $R_{s}$ may increase or conversely the electrically active sheet carrier density $\left(N_{s}\right)$ may decrease when a phosphorus implantation is combined with VEI, which is contrary to the results presented in Fig. 5 for the $700-900^{\circ} \mathrm{C}$ temperature range. In the current study the phosphorus dose was high enough to amorphize the first $5 \mathrm{~nm}$ of the sample, rendering the effects of the VEI redundant. The peak concentration of the phosphorus profile would reside within the amorphous layer and therefore electrical activation would occur via solid-phase epitaxy. Any direct influence of the VEI on phosphorus activation would only occur for the phosphorus residing beyond the amorphous 5nm region. Furthermore, any variation in depth of the amorphous layer, with or without VEI, would be also reflected in a variation of $N_{s}$. TEM analysis was used to verify the amorphous layer depth for samples implanted with phosphorus, with and without VEI, and these are presented within Fig. 6. The small difference in the amorphous layer depth does not account for level of increased $N_{s}\left(\sim 4 \mathrm{x}\right.$ at $\left.750^{\circ} \mathrm{C}\right)$. We further note that the dark contrast band, present beneath the amorphous layer in the sample implanted with only phosphorous, is absent when the phosphorus implant is combined with VEI. This is indicative of a change in sample density which infers a reduction in silicon interstitial concentration below the amorphous/crystalline (A/C) interface.

The evidence presented here allows us to suggest that phosphorus atoms cluster with interstitial defects beyond the A/C interface. Further, the demonstrated improvements in electrical activation (Fig.5) are due to a reduction of Phosphorous Interstitial Clusters (PICs) within the tail region of the phosphorus distribution due to 
the initial presence of vacancies generated by the VEI. This is a result of the reduction of the density of interstitials at the $\mathrm{A} / \mathrm{C}$ interface and/or the inhibition of the PIC evolution through a vacancy - interstitial annihilation mechanism. This hypothesis supports the studies performed by Uematsu et al. who found that it was only possible to accurately simulate phosphorus diffusion after implementation of a phosphorous - interstitial clustering model.

\section{Conclusion}

In this report we describe the impact of a Vacancy Engineering Implant (VEI) on the diffusion and activation of ultra-shallow phosphorus in silicon, implemented via $2 \mathrm{keV}$ $\mathrm{P}+$ ion implantation to a dose of $1 \times 10^{15} \mathrm{~cm}^{-2}$. Using Variable Energy Positron Annihilation Spectroscopy (VEPAS), we have confirmed the presence of excess vacancy-type defects following thermal annealing at $300^{\circ} \mathrm{C}$, during which local vacancy-interstitial annihilation occurs. The measured net excess vacancy concentration is in agreement with that obtained via Monte-Carlo simulation. The post-implantation diffusion of phosphorus is significantly retarded when VEI is applied (relative to the sample without VEI), as one might expect for an interstitial diffuser. Further, the activation of phosphorus is enhanced for the VEI sample compared to that without a VEI, a somewhat surprising result given that phosphorus is well-known to cluster with vacancy-type defects. This leads us to postulate that a combination of a high dose, low energy phosphorus implantation, combined with a VEI leads to the reduction of phosphorus - interstitial clusters within the tail region (i.e. beyond the amorphous/crystalline interface) of the as-implanted phosphorus distribution. 


\section{References}

[1] International Roadmap for Semiconductors 2008, http://www.itrs.net

[2] W. Hofker, H. Werner, D Oosthoek and H. de-Grefte, App. Phys. 2, 265 (1973)

[3] N. Cowern, K. Janssen and H. Jos, J. Appl. Phys. 68, 6191 (1990)

[4] N. Cowern, A. Smith, B. Colombeau, R. Gwilliam, B. Sealy and E. Collart, IEDM Technical Digest 2005 (IEEE, Piscataway, NJ), 39.11

[5] E. Roth, O. Holland, V. Venezia and B. Nielsen, J. Elec. Mat. 26, 11, 1349 (1997)

[6] A. Smith, B. Colombeau, R. Gwilliam, N. Cowern, B. Sealy, M. Milosavljevic, E. Collart, S. Gennaro, M. Bersani, M. Barozzi, Mat. Sci. Eng. B, 124-125, 210 (2005) [7] A. P. Knights and P. G. Coleman Def. and Diff. Forum 183-5 41-52 (2000). [8] A. Mazzone, Phys. Stat. Sol. (a) 95, 149 (1986)

[9] N. B. Chilton and P. G. Coleman, Meas. Sci. Technol. 6 53-59 (1995).

[10] P.G. Coleman, A. P. Knights and M. J. Anc, J. Appl. Phys. 93, 698-701 (2003).

[11] A. P. Knights, F. Malik and P. G. Coleman, Appl. Phys. Lett. 75, 466-8 (1999).

[12] G.C. Aers, in: P.J. Schultz, G.R. Massoumi, G.R. Simpson (Eds.), Positron Beams for Solids and Surfaces, AIP conf. proc. 218, New York, 1990, p. 162.

[13] A. P.Knights, P. J. Simpson, L. B. Allard and J. L. Brebner, J. Appl. Phys. 79 $9022(1996)$.

[14] P. G. Coleman and C. P. Burrows, Phys. Rev. Lett., 98, 265502 (2007).

[15] A. Smith, N. Cowern, R. Gwilliam, B. Sealy, B. Colombeau et. al. App. Phys. Lett. 88, 082112 (2006)

[16] M. Uematsu, Jap. J. App. Phys. 38, 6188 (1999) 
Figure Captions.

Figure 1 - Schematic representation of the VEI with respect to the SOI structure using a Monte Carlo simulation (a) combined with equation 1 to present the estimated net excess vacancy (open squares) and silicon interstitial (open circles) distributions. Diffraction patterns where taken from the indicated locations (arrows do not correspond to the exact location) to examine the crystallinity of silicon structure after the VEI (b-e).

Figure 2 - Normalized S-parameter versus implanted positron energy for $110 \mathrm{~nm}$ overlayer SOI. Closed squares - unimplanted SOI; Open squares - SOI implanted with $\mathrm{Si}^{+}$ions at an energy of $300 \mathrm{keV}$ and a dose of $1.5 \times 10^{15} \mathrm{~cm}^{-2}$; Open triangles identical implantation and subsequent anneal at $300^{\circ} \mathrm{C}$; Closed diamonds - identical implantation and subsequent anneal at $300^{\circ} \mathrm{C}$. The lines are fits to the data obtained with the POSTRAP routine [12].

Figure 3 - Open volume defect concentrations measured via VEPAS as a function of anneal temperature. The second y-axis illustrates the equivalent total areal density of defects assuming a 'box-like' distribution where the width of the distribution is fixed at the width of the silicon overlayer (110nm).

Figure 4 - SIMS analysis of a $2 \mathrm{keV} \mathrm{P}$ implant to a dose of $10^{15} \mathrm{~cm}^{-2}$ as function of anneal temperature. (a)and (b) present the $\mathrm{P}$ profiles without and with a VEI, respectively. 
Figure 5 - Sheet resistance and Hall measurements of the phosphorus layers as a function of anneal temperature, with (open squares) and without (open circles) a VEI, presenting $\mathrm{R}_{\mathrm{s}}$ (a), carrier density (b) and Hall mobility (c).

Figure 6 - X-TEM analysis of the surface silicon over layer in the SOI structure, illustrating the amorphous surface layers generated via the phosphorus implant (a) and the phosphorus + VEI (b). 
Figure 1.

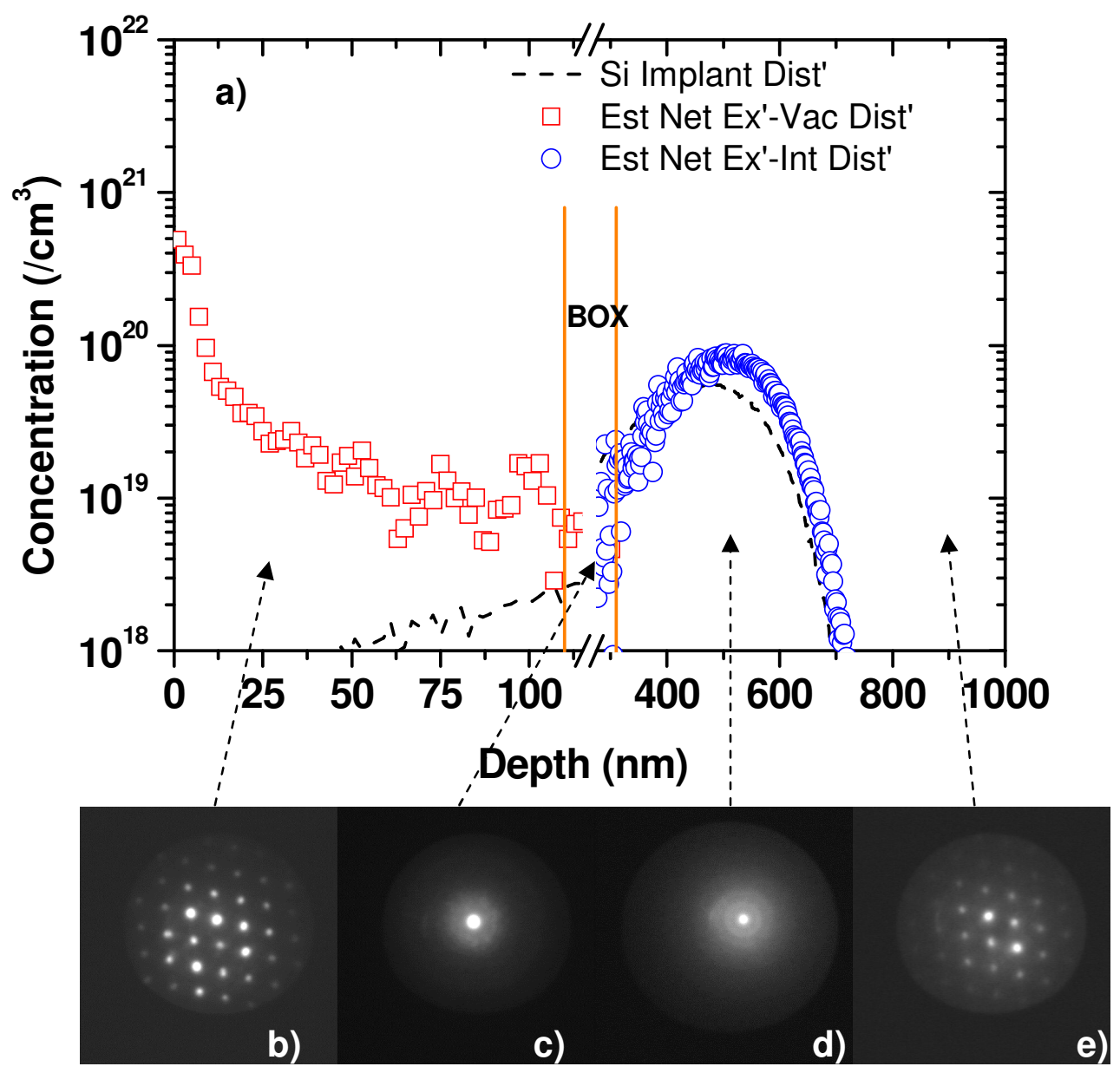


Figure 2.

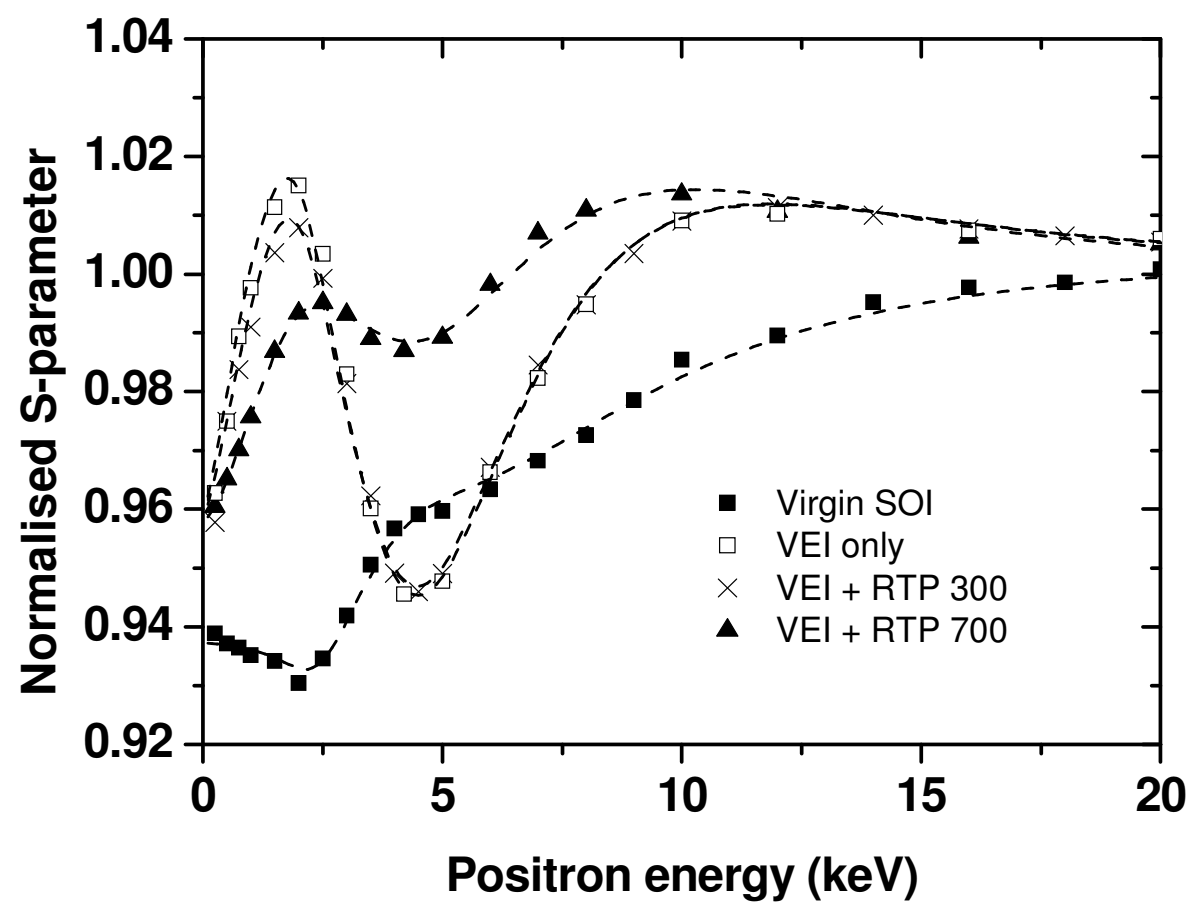


Figure 3.

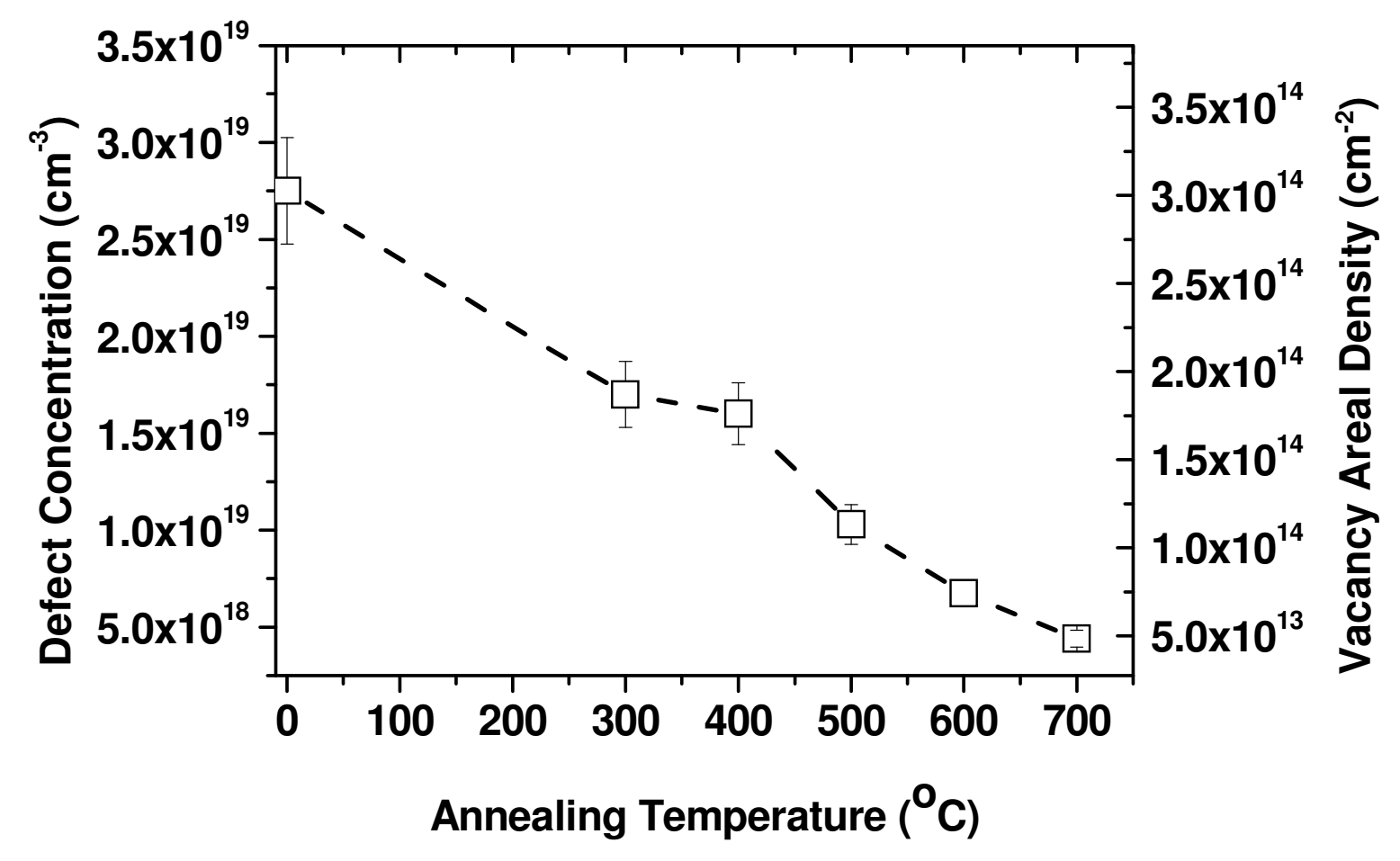


Figure 4.

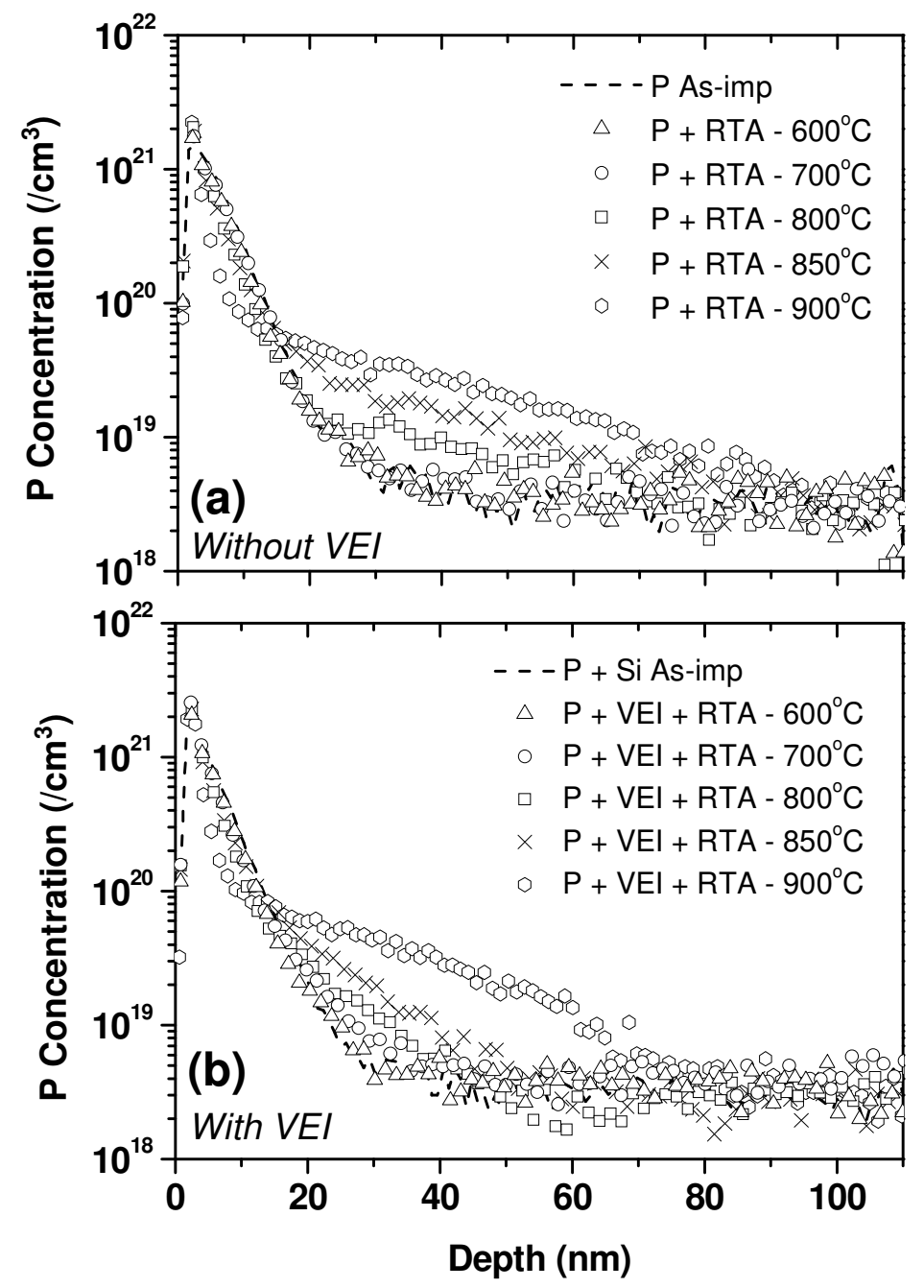


Figure 5.

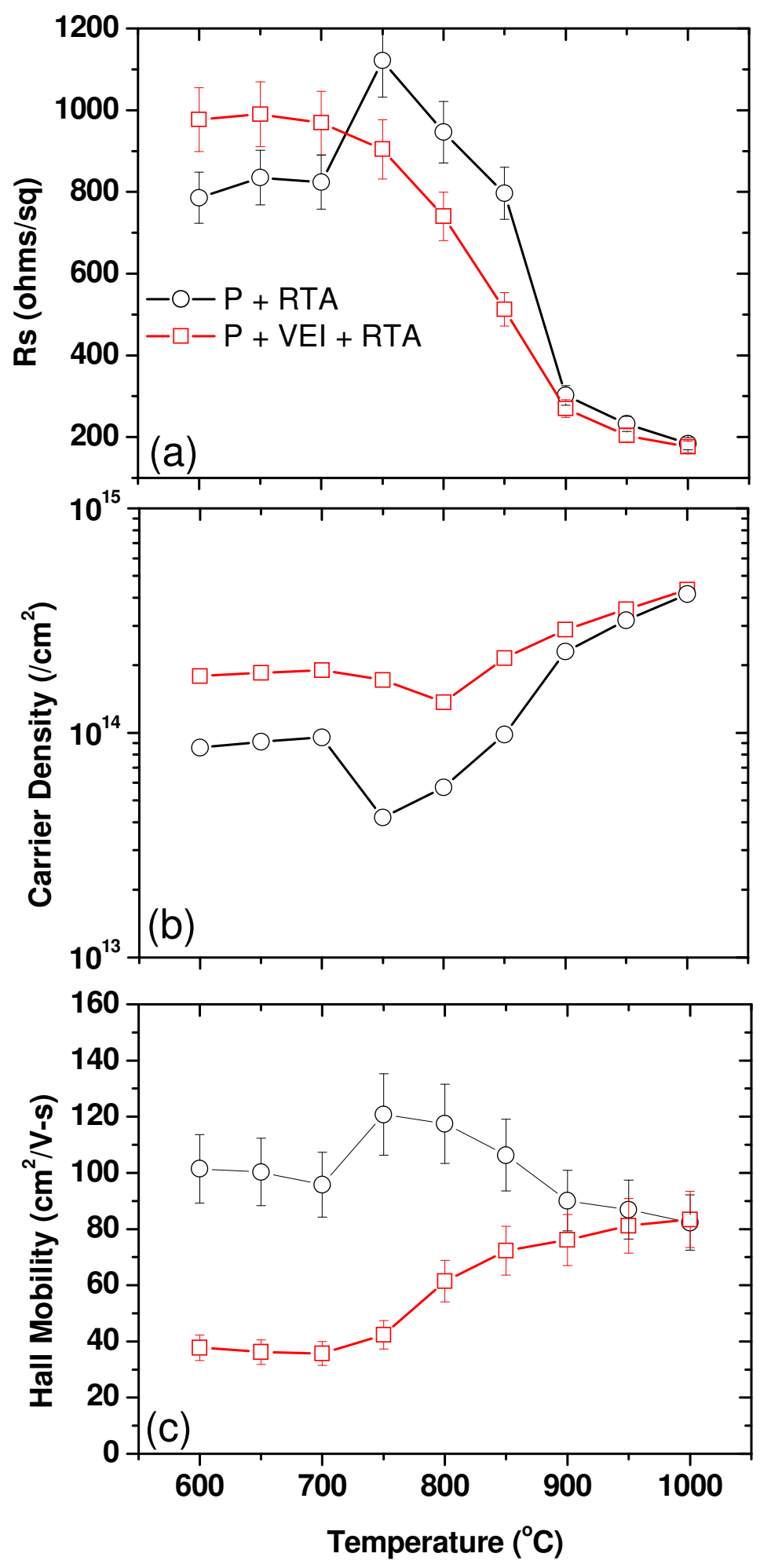


Figure 6.
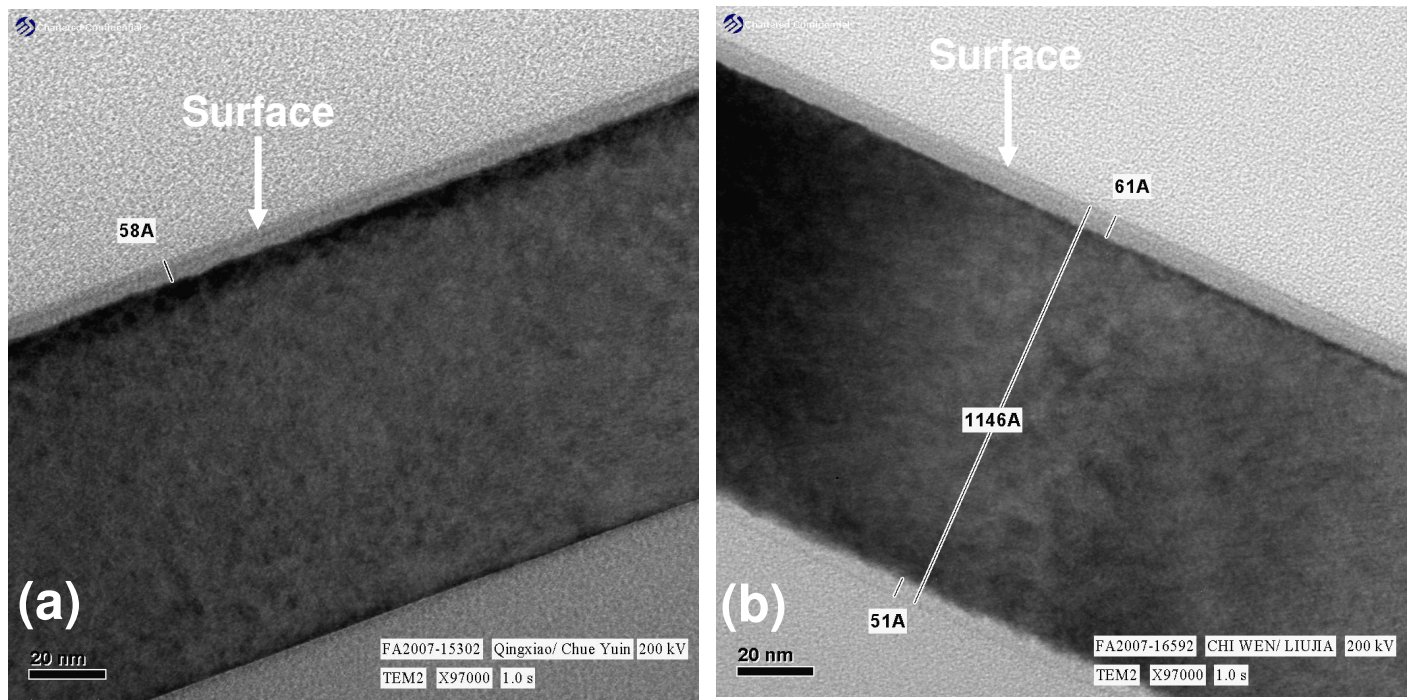